\title{
Structure, Bose-Einstein condensation, and superfluidity of two-dimensional confined dipolar assemblies
}

\author{
Piyush Jain, ${ }^{*}$ Fabio Cinti ${ }^{\dagger}$ and Massimo Boninsegni \\ Department of Physics, University of Alberta, Edmonton, Alberta, Canada \\ (Received 6 April 2011; revised manuscript received 12 May 2011; published 29 July 2011)
}

\begin{abstract}
Low-temperature properties of harmonically confined two-dimensional assemblies of dipolar bosons are systematically investigated by Monte Carlo simulations. Calculations carried out for different numbers of particles and strengths of the confining potential yield evidence of a quantum phase transition from a superfluid to a crystal-like phase, consistently with what is observed in the homogeneous system. It is found that the crystal phase nucleates in the center of the trap, as the density increases. Bose-Einstein condensation vanishes at $T=0$ upon entering the crystalline phase, concurrently with the disappearance of the superfluid response.
\end{abstract}

DOI: 10.1103/PhysRevB.84.014534

PACS number(s): 05.30.Jp, 03.75.Hh, 31.15.xk, 64.60.an

\section{INTRODUCTION}

Bose-Einstein condensation (BEC) and superfluidity (SF) are intimately connected quantum many-body phenomena, ${ }^{1}$ both underlain by long cycles of exchange of identical particles. ${ }^{2}$ In three dimensions BEC and SF appear simultaneously, and become almost synonymous when interactions among particles are weak, a condition normally experimentally attained in trapped ultracold gases. ${ }^{3,4}$ In that limit, the fractions of the system that are superfluid and Bose condensed both approach $100 \%$ at the temperature $T \rightarrow 0$. This provides a major justification for theoretical studies based on mean-field techniques. ${ }^{5,6}$

On the other hand, the effect of strong interparticle interactions on SF and BEC is very different. A chief example is provided by liquid ${ }^{4} \mathrm{He}$, which approaches the $100 \%$ superfluid limit as $T \rightarrow 0$, while its condensate is greatly depleted; indeed, theoretical ${ }^{7,8}$ and experimental ${ }^{9,10}$ estimates for the condensate fraction in superfluid ${ }^{4} \mathrm{He}$ yield a value in the neighborhood of 7\%-8\%, at $T=0$. Furthermore, no theoretical understanding currently exists of the relationship, if any, between the local values of the superfluid and condensate fractions. Broadly speaking, the most quantitative aspects of the relationship between these two quantities remain to be further elucidated.

Impressive advances in the trapping and manipulation of ultracold atoms appear to offer a pathway to a more in-depth investigation of this relationship, by making it feasible to create in the laboratory "artificial" many-body systems characterized by interactions of variable strength and shape. Thus, one may smoothly interpolate between weakly and strongly interacting regimes, as well as explore the effect on SF and BEC of interactions not (easily) realized in ordinary condensed matter systems.

In recent times, attention has turned to the case of dipolar bosons, further bolstered by the realization of Bose-Einstein condensation of chromium. ${ }^{11}$ The long-range and anisotropic nature of the dipole-dipole interaction leads to many fascinating phenomena. ${ }^{12}$ The conceptually simplest scenario is that of an assembly of dipolar bosons confined to two dimensions, with their dipole moments aligned by an external (electric or magnetic) field directed perpendicular to the confinement plane. In this case, the interaction between any two particles is purely repulsive, proportional to the inverse cubic power of the distance between them.

Recently, it was shown that a mixture of equal-mass dipolar isotopes, in such a configuration, demixes at finite temperature due to quantum statistical effects. ${ }^{13}$ A promising direction for implementing trapped dipolar assemblies is through the use of Rydberg-excited atoms. The large induced dipole moments of such systems may facilitate the realization of solidlike phases. ${ }^{14,15}$

The phase diagram of a homogeneous system of purely repulsive dipolar bosons in two dimensions has been explored by Monte Carlo simulations. ${ }^{16-18}$ These works have yielded evidence of a quantum phase transition at $T=0$ between a superfluid and a crystal phase, but the quantitative characterization of such a phase transition, e.g., its location and the width of the coexistence region, has proven surprisingly difficult, due to the long-range nature of the interaction. Theoretical arguments ${ }^{19}$ have been put forth to the effect that a conventional first-order quantum phase transition ought not occur in this system, as the long-ranged nature of the interactions renders the coexistence of two phases of different densities, separated by a single macroscopic interface, energetically unfavorable. Rather, an "emulsion" should form, consisting of (relatively) large solid domains embedded in the superfluid. Such a scenario has not yet been observed in computer simulations, presumably due to the need to study systems of size not currently attainable.

The superfluid transition at finite temperature has been predicted to be compatible with the Berezinskii-KosterlitzThouless theory. ${ }^{20}$ And, while no evidence has been seen of a commensurate supersolid phase, ${ }^{18,19,21}$ its presence has not been ruled out yet (it is in fact predicted to occur in a system of dipolar bosons in the so-called Rydberg-blockaded regime $^{22}$ ).

It is expected that many of the outstanding issues will soon be elucidated by experiments on spatially confined assemblies of dipolar particles. Two-dimensional confinement can be achieved in the laboratory by means of a harmonic trap in the transverse direction, of frequency $\omega_{z}$. Clearly, the system must also be confined in the remaining two dimensions, as the interactions are purely repulsive, and this is accomplished by means of a second, in-plane harmonic trap, of frequency $\omega \ll \omega_{z}$, 
realizing the so-called pancake geometry. The number of particles that can be currently trapped is typically of the order of a few thousands, which means that the physics of such a system is strongly influenced by its finite size. Thus, theoretical studies of finite two-dimensional dipolar systems in a harmonic potential seem especially opportune and timely, in order to guide in the interpretation of experiments aimed at inferring bulk properties.

However, a finite system of dipolar bosons is of interest in its own right, chiefly because novel fundamental understanding of the relationship between BEC and SF can be gained. Indeed, theoretical work ${ }^{23,24}$ on clusters of ${ }^{4} \mathrm{He}$ has shown that superfluid and condensate fractions can be meaningfully and rigorously defined for systems comprising as few as $N=70$ particles. One should note that, for the two-dimensional systems considered in this work, because the system is finite, reduced dimensionality does not imply absence of BEC at finite temperature. ${ }^{25,26}$ Moreover, while the superfluid and condensate are uniform in the bulk system, owing to translational invariance, in confinement they can display nontrivial local variations. Of particular import seems the comparison of the local superfluid and condensate fractions, which can lead to original insight into their interplay.

Previously, Lozovik et al. ${ }^{27}$ and more recently Pupillo et al., ${ }^{15}$ have studied harmonically confined dipolar systems of relatively small size (up to $N=40$ particles). In this paper, we investigate by means of Monte Carlo simulations the low-temperature phase diagram of dipolar Bose assemblies comprising up to $N=1000$ particles, a number which appears relevant to current (or planned) experiments.

The purpose of this work is twofold. On the one hand, we compare the ground-state phase diagram of the trapped system against the predictions made in Refs. 16-18 for the bulk case. Our aim is to make contact between the theoretical phase diagram of the bulk and the quantitative information that can be provided by experiments on finite-size systems, comprising a relatively small number of particles. Second, we compute both global and local densities of the superfluid and Bose-Einstein condensate, based on numerically unbiased estimators, and study how the two quantities evolve as the strength of the interactions in the system increases (as measured by the density in the middle of the trap). To the best of our knowledge, this is the first system for which both the local superfluid and condensate densities are computed simultaneously for a strongly interacting many-body system, based on a methodology free from approximations.

This paper is organized as follows. In Sec. II we introduce the model Hamiltonian for a two-dimensional system of dipolar Bose particles harmonically confined, and discuss the relevant observables pertinent to structure, Bose-Einstein condensation, and superfluidity. We illustrate our results in the following three sections, Sec. III focusing on the structure of the system with respect to system size and in-trap mean interparticle distance, Sec. IV focusing on the superfluidity and condensation, notably both global and local properties, and finally Sec. V discussing the ground-state phase diagram, as well as the momentum distribution. We outline our conclusions in Sec. VI.

\section{MODEL AND METHODOLOGY}

We consider a two-dimensional system comprising $N$ bosons of mass $m$, confined by a harmonic potential of frequency $\omega$. Particles then interact via a purely repulsive, pairwise dipolar potential, given by $v(r)=D / r^{3}, r$ being the distance between the particles. The simplest way of realizing such an interaction is with particles possessing an electric (magnetic) dipole moment, aligned along the transverse direction by an applied electric (magnetic) field. ${ }^{16}$ The quantum-mechanical many-body Hamiltonian is given in dimensionless form by

$$
\hat{H}=-\frac{1}{2} \sum_{i}^{N} \nabla_{i}^{2}+\Gamma \sum_{i}^{N} \mathbf{r}_{i}^{2}+\sum_{i<j}^{N} \frac{1}{\left|\mathbf{r}_{i}-\mathbf{r}_{j}\right|^{3}},
$$

where $\mathbf{r}_{i}$ is the position of the $i$ th particle, and where we have introduced length and energy scales respectively as $r_{\circ}=m D / \hbar^{2}$ and $\epsilon_{\circ}=\hbar^{2} / m r_{\circ}^{2}$. The trap strength parameter is $\Gamma=(1 / 2)\left(r_{\circ} / L\right)^{4}, L=\sqrt{\hbar / m \omega}$ being the oscillator length. It is also useful to introduce a reduced temperature $T^{*}=$ $k_{B} T /(\hbar \omega)$, with $T$ expressed in units of $\epsilon_{\circ}$.

We investigate the low-temperature properties of this finite system by means of Monte Carlo simulations. We consider systems with particle numbers $N$ ranging from 20 to 1000 , and trap strengths $\Gamma=5 \times 10^{-2}-5 \times 10^{6}$. We use the worm algorithm, in the continuous-space path integral representation. ${ }^{8,28}$ The methodology is numerically exact, errors being only statistical in character and reducible to an essentially negligible size with a relatively modest computer time expenditure. Technical details of the calculation are the same as in other studies; ${ }^{29-31}$ the use of the dipolar interaction in (1) entails no particular difficulty. All of our quoted ground-state results, with their uncertainties, are obtained by extrapolating our numerical estimates to the limit of temperature $T=0$. In general, we have observed that the structural and energetic properties of the assemblies considered here do not change, within the statistical error of our calculations, if the temperature $T^{\star}$ is $\lesssim 0.1$, below which the system may be regarded as essentially in its ground state. As customary in this type of numerical study, we have also carried out extrapolation of our estimates to the limit of vanishing time step. ${ }^{31}$ As a typical example, Fig. 1 shows the total (potential plus kinetic) energy per particle as a function of (imaginary-time) step size $\Delta \tau$. As the step size is made sufficiently small, the energy converges to a fixed value.

As mentioned above, one of our aims is to make contact between the ground-state properties of the finite assembly considered here and those of the same system in the bulk limit, where the physics is determined solely by the mean interparticle distance $r_{s}=1 / \sqrt{\bar{\rho} r_{\circ}^{2}}, \bar{\rho}$ being the particle density. For the trapped system, the density is not uniform, but rather is a function $\rho(r)$ of the radial distance from the center of the trap. Near the center of the trap, the physical behavior of the finite system ought to resemble most closely that of the bulk; thus, we associate a value of $r_{s}$ with the trapped system by extrapolating to $r \rightarrow 0$ the behavior of the function

$$
r_{s}(r)=\frac{1}{r_{\circ} \sqrt{\rho(r)}} .
$$

Details of the extrapolation are given below. 


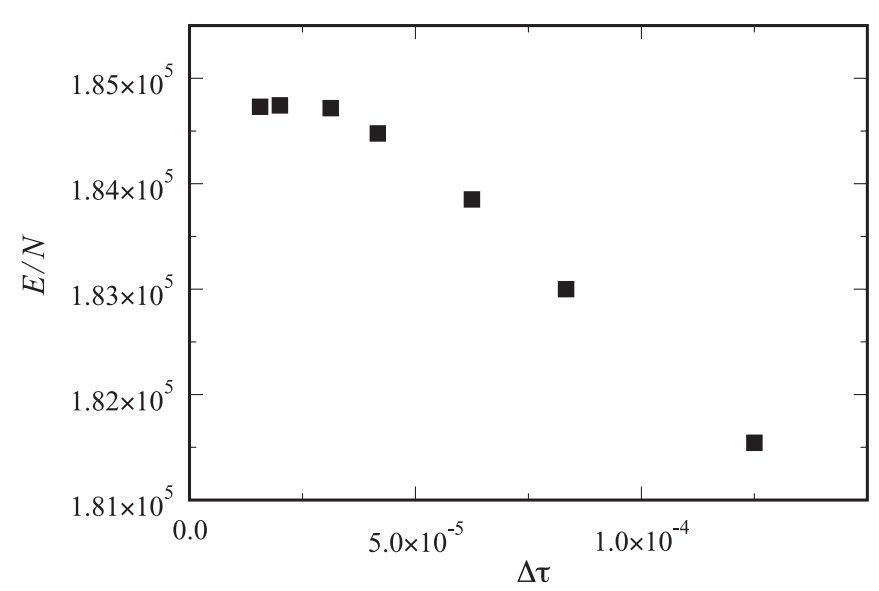

FIG. 1. Energy per particle as a function of (imaginary-time) step size for worm algorithm simulations with $\Gamma=5 \times 10^{6}, N=100$, and inverse temperature $\beta=1 / 500$ (where $k_{B}=1$ ). In this case numerical convergence is found for $\Delta \tau=\beta / M \lesssim 2 \times 10^{-5}$ where $M$ is the number of time steps. Errors are smaller than the symbol size.

In addition to the radial density $\rho(r)$, we compute the local superfluid $\left[\rho_{s}(r)\right]$ and condensate $\left[\rho_{c}(r)\right]$ densities. The average superfluid density $\rho_{s}$ is computed using the area estimator. ${ }^{24}$ The local superfluid density is computed by means of an estimator first proposed by Kwon et al.: ${ }^{32}$

$$
\frac{\rho_{s}(r)}{\rho(r)}=\frac{4 m^{2} T\langle A A(r)\rangle}{\beta \hbar^{2} I_{c}(r)},
$$

where $\beta=1 / T$ (we set the Boltzmann constant equal to 1 ), $\langle\cdots\rangle$ stands for thermal average, $A$ is the area swept by the many-particle world lines in the $x-y$ plane, and where $A(r)$ and $I_{c}(r)$ are respectively the contributions to $A$ from paths in a shell of radius $r$ and $I_{c}(r)$ its classical moment of inertia.

The estimation of the condensate fraction is carried out based on the ideas expounded in Refs. 33 and 34. Specifically, we compute by Monte Carlo simulations the angularly averaged one-body density matrix

$$
\rho_{0}\left(r, r^{\prime}\right)=\left\langle\hat{\psi}^{\dagger}\left(r^{\prime}\right) \hat{\psi}(r)\right\rangle,
$$

where $\hat{\psi}$ and $\hat{\psi}^{\dagger}$ are the usual Bose field operators. The matrix $\rho_{0}$ has positive-definite eigenvalues, the largest of which is the average number of particles in the maximally occupied natural orbital (condensate), which is the corresponding eigenfunction. ${ }^{35}$ The assumption is made here that the condensate should be cylindrically symmetric. As we are only interested in its largest eigenvalue $N_{0}$ here, and its associated natural orbital $\phi_{0}$, we solve the eigenvalue equation for $\rho_{0}$ iteratively (numerically), using the power method. The condensate fraction is then given by $N_{0} / N$ with corresponding local condensate density given by $\rho_{c}(r)=\left|\phi_{0}(r)\right|^{2}$.

\section{STRUCTURE}

We begin by discussing the structural properties of the system as a function of the density of particles in the trap, in the $T \rightarrow 0$ (ground-state) limit. Numerical studies of the ground-state phase diagram of a two-dimensional homogeneous system of dipolar bosons have yielded evidence of a superfluid-to-crystal quantum phase transition. ${ }^{16-18}$ The precise determination of the location and width of the coexistence region has so far proven rather challenging. Büchler et al. ${ }^{16}$ place it within the relatively large range $0.045 \leqslant r_{s} \leqslant$ 0.065 , and other estimates ${ }^{17,18,20}$ are in general agreement. The question is what remnants, if any, of such a phase transition can be observed on a finite system.

A first, general comment is in order: one must exercise caution when referring to phase transitions in finite-size (mesoscopic) systems. It is well known that, although such systems may exhibit "phaselike behavior," the coexistence of two phases, which is typical of a first-order phase transition, is energetically disfavored by the presence of an interface separating the two phases. Thus, in a finite-size system, especially of the size treatable in any realistic numerical simulation, phase transitions are smoothed out. ${ }^{36}$ To some extent, therefore, even though we shall make use of this terminology, any reference to "phase" must be understood qualitatively. As well, obviously the fact that the system is confined by a harmonic trap can be expected to influence its physics qualitatively, especially at the border where particle localization is enhanced by the steepness of the confining harmonic potential.

Figure 2 shows instantaneous configurations (i.e., particle world lines) of a system comprising $N=200$ dipolar bosons, for different values of the trap strength $\Gamma$, increasing from (a) to (d). These results correspond to temperatures sufficiently low that the system may be regarded as essentially in its ground state. These snapshots offers visual, qualitative insight into the evolution of the system as the density inside the trap is increased. The presence of a featureless fluid phase at low density, and the emergence of crystalline order at high density, with particles arranged on a triangular lattice near the center of the trap, are clear in Fig. 2. Specifically, at high density (i.e., greater trap strength) particle world
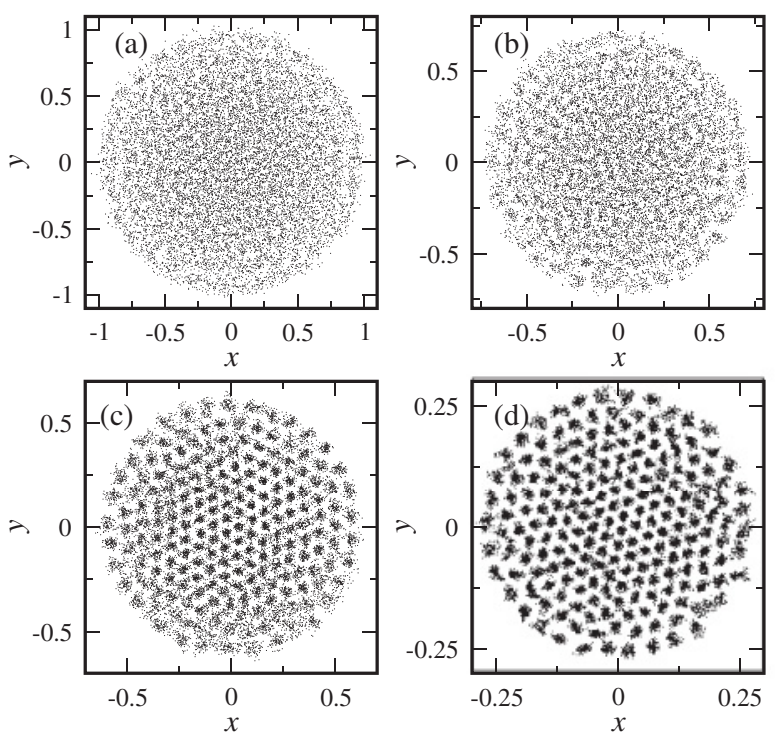

FIG. 2. Snapshots of many-particle world lines for a trapped system comprising $N=200$ bosons, in the low-temperature limit (i.e., $T \rightarrow 0$ ), for values of the trap strength (a) $\Gamma=1 \times 10^{4}$; (b) $\Gamma=5 \times 10^{4}$; (c) $\Gamma=1 \times 10^{5}$; (d) $\Gamma=5 \times 10^{6}$. 

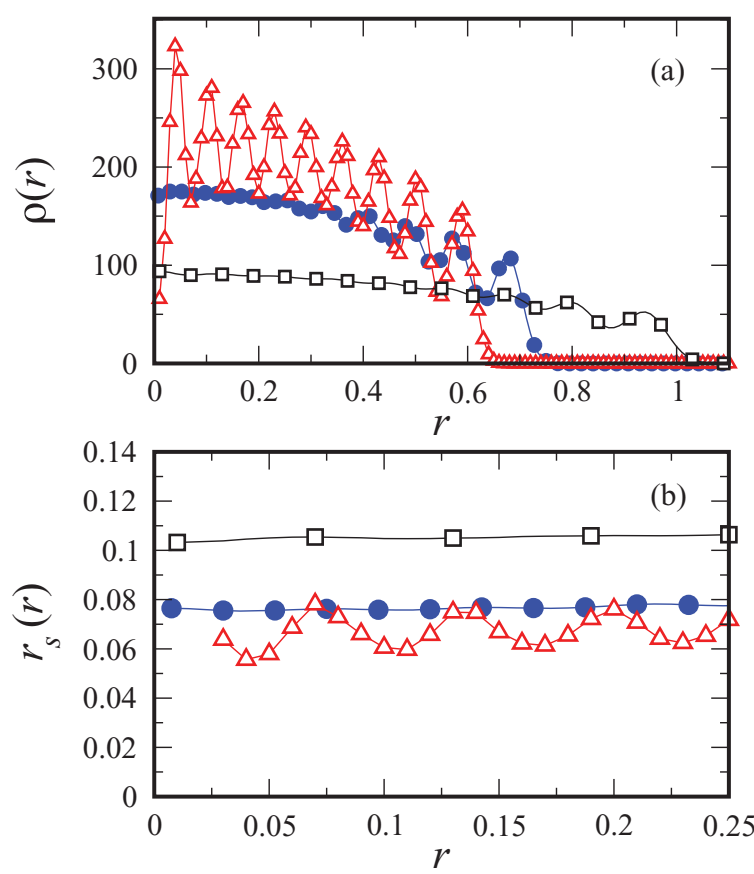

FIG. 3. (Color online) Density profiles for a dipolar system comprising $N=200$ particles, for values of the trap strength $\Gamma=$ $1.0 \times 10^{4}$ (squares), $\Gamma=5.0 \times 10^{4}$ (circles), and $\Gamma=1.0 \times 10^{5}$ (triangles). All values of $\Gamma$ are in units of $\epsilon_{\circ}$. (a) Local density $\rho(r)$. (b) Corresponding local value of the interparticle distance $r_{s}(r)$, defined in Eq. (2). Statistical errors are smaller than symbol sizes.

lines become localized, and exchanges of (indistinguishable) particles suppressed, as shown for the largest trap strength in Fig. 2(d).

More quantitative information is offered by plots such as those of Fig. 3, which shows radial density profiles for a trapped system comprising $N=200$ particles, for different values of the confining strength $\Gamma$ of the trapping potential. Figure 3(a) shows the local density of particles $\rho(r)$. At low density (i.e., low values of $\Gamma$ ), $\rho(r)$ is essentially constant near the center, whereas at the edge of the trap, where the confining potential dominates the energetics, a shell structure forms. On the other hand, at high density the dipole-dipole interaction energy dominates in the center of the trap, where $\rho(r)$ displays oscillations that are consistent with a regular arrangement of particles.

On the expectation that the physical behavior of the system should mimic that of the bulk near the center of the trap, we may compare results for a trapped system with those in the thermodynamic limit by attributing an effective value of $r_{s}$ to the confined system. As explained above, we do so by taking the value of $r_{s}(r)$ at $r=0$ (i.e., the center of the trap), or, in the case of oscillatory behavior [e.g., triangles in Fig. 3(b)], we take the value of $r_{s}(r)$ averaged over a few periods of oscillation near the center. Figure 3 shows typical results for $r_{s}(r)$, as defined as in (2). We estimate the uncertainty in the values of $r_{s}$ determined in this way, quoted in the remainder of the paper, to be of the order of $5 \%$.

For a system with $N=200$ particles, the results shown in Fig. 3 suggest that a transition from a uniform liquid phase to a crystalline one takes place for $r_{s}$ roughly below $r_{s} \lesssim 0.06$.
A more precise assessment of the density region within which the transition takes place can be obtained by studying superfluidity and Bose-Einstein condensation; we come back to this later on.

In order to establish a more quantitative connection between the physics of the trapped assembly and that of a bulk system, we compute the pair correlation function in a circular region of radius $R$, centered at $r=0$. Such a "local pair correlation function" inside this region, $g_{R}(r)$, has the normalization

$$
2 \pi \rho_{R} \int_{0}^{R} d r r g_{R}(r)=N_{R}-1,
$$

where $N_{R}$ is the average number of particles in the bounded region, and the corresponding average density is given by $\rho_{R}=N_{R} / \pi R^{2}$.

Figure 4 shows the computed $g_{R}(r)$ for trapped systems with different numbers of particles, specifically $N=100$ (diamonds), 200 (squares), and 1000 (circles), in the $T \rightarrow 0$ limit. Results are shown for different trap strengths $\Gamma$, adjusted to yield a value of $r_{s}$ in the middle of the trap around 0.03 [Fig. 4(a)] and 0.085 [Fig. 4(b)]. Here, we took $R=0.13$ and 0.3 , respectively, i.e., values smaller than the radius at which
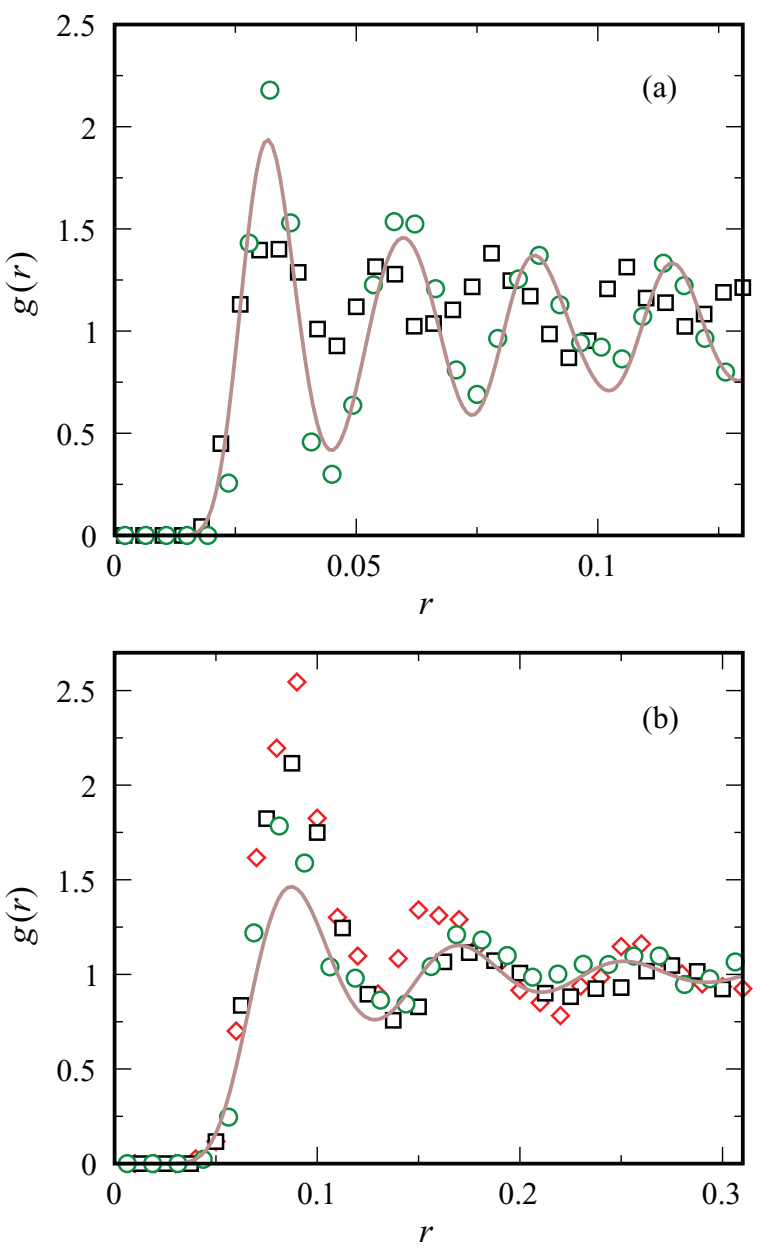

FIG. 4. (Color online) Local pair correlation function for trapped dipolar bosons with $N=100$ (diamonds), 200 (squares), and 1000 (circles), and in the bulk case (solid lines). The interparticle distance $r_{s}$ in the middle of the trap is $r_{s}=0.03$ (a) and $r_{s}=0.085$ (b). Statistical errors are of the order of the symbol size. 
the density goes to zero in the trap. For $r_{s}=0.03$, the ground state of the bulk system is crystalline; a superfluid for $r_{s}=$ 0.085 . Both figures also show (solid lines) the corresponding pair correlation functions for the bulk system with the same values of $r_{s}$, also computed in the $T \rightarrow 0$ limit (a separate calculation was performed for this case, using standard bulk methodology, with periodic boundary conditions).

The first observation is that in both cases, on increasing the number of particles, $g_{R}(r)$ approaches that of the bulk system, as expected. Moreover, the finite size of the system, combined with the fact that the trap is harmonic, enhances particle localization and therefore strengthens crystalline order. Results shown in Fig. 4(a) correspond to $r_{s}=0.03$ in the middle of the trap; $g_{R}(r)$ displays marked oscillations for both $N=200$ and 1000 particles. However, for the smaller system size the height of the peaks is lower, and the distance between successive peaks is also different from that observed in the bulk. This suggests that, although the physics of the system is solidlike in both cases, for the smaller system it is substantially influenced by the trap, whereas with five times more particles it is already rather close to that of the bulk. Indeed, the pair correlation function in the center of the trap is virtually indistinguishable from that of the bulk system beyond the first peak, within our statistical errors. It displays the pronounced oscillations that one expects for a crystalline phase.

Figure 4(b) shows results for the case $r_{s}=0.085$. Here too, the local pair correlation function has a noticeable dependence on the size of the system. However, in this case the distance between the peaks is roughly the same in all cases, i.e., the influence of the edge on the physics of the system at the center of the trap is less significant than in the case previously discussed (not surprisingly, as the trap is weaker). As the size of the system is increased from $N=100$ to 1000 particles, the most significant change is the height of the first peak of $g_{R}(r)$, which decreases by roughly $30 \%$. Beyond the first peak, the pair correlation functions for $N=200$ and 1000 are essentially indistinguishable from one another, as well as from that of the bulk system (again within the statistical uncertainties of our calculation), and are consistent with liquidlike behavior.

Figure 5 shows snapshots as in Fig. 2 for $N=1000$ at low temperature, corresponding to the two values of $r_{s}$ shown in Fig. 4. For $r_{s}=0.03$, an orderly arrangement of atoms in a triangular lattice occurs in the center of the trap, confirming the picture given by consideration of the local pair correlation function. It is important to note, however, that exchanges still take place, primarily in the peripheral disordered region, where the system goes from bulk crystal (center of the trap) to the arrangement on concentric rings characteristic of the surface region.

As mentioned above, the crystal-to-superfluid transition in the bulk is expected to take place roughly in the $0.045 \leqslant r_{s} \leqslant$ 0.065 interval. The results obtained in this work show that a harmonically trapped system comprising at least $N=1000$ particles ought to allow one to observe experimentally some of the relevant physics. On the other hand, the behavior of systems comprising just a few hundred particles is still greatly influenced by the confining potential, in the same range of $r_{s}$. A more quantitative discussion of the physics of the trapped system, and its connection to the bulk superfluid-to-insulator
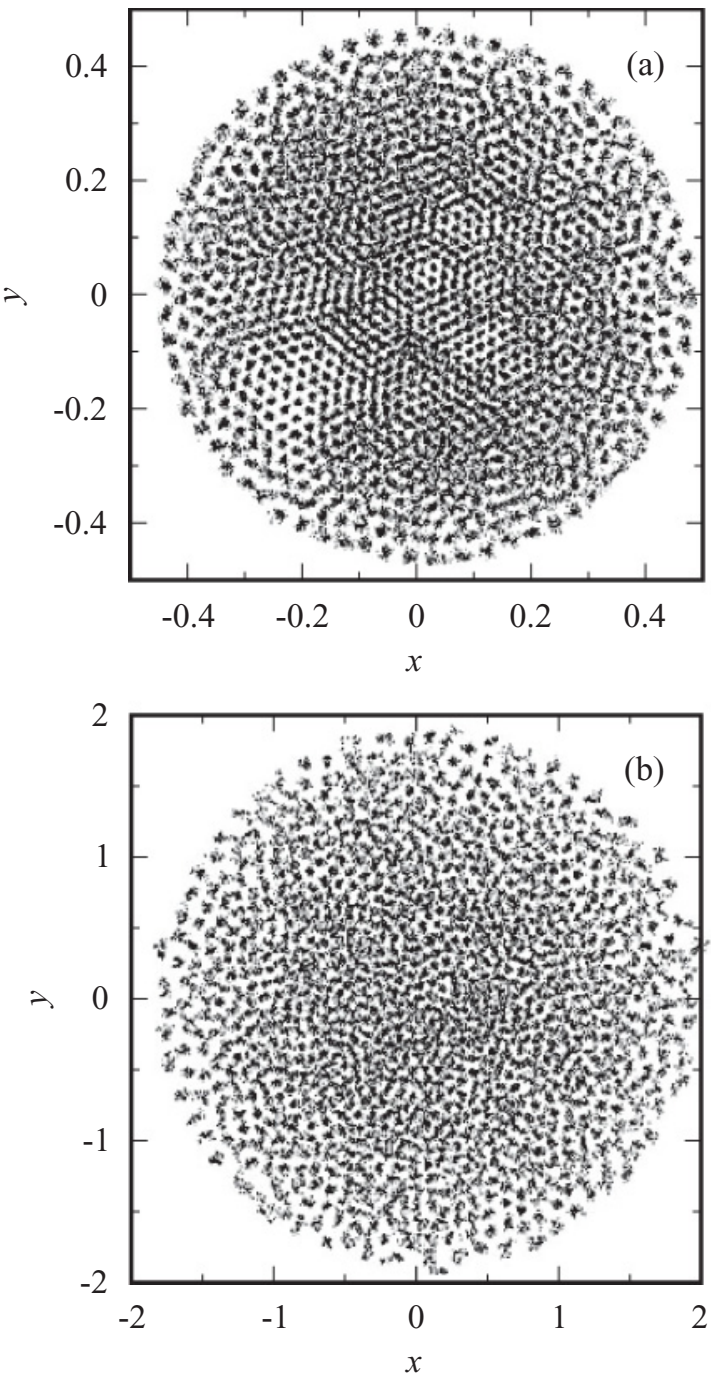

FIG. 5. Snapshots of many-particle world lines for a trapped system comprising $N=1000$ bosons, in the low-temperature limit (i.e., $T \rightarrow 0$ ), for values of the trap strength $\Gamma$ yielding an interparticle distance in the middle of the trap equal to (a) $r_{s}=0.03$; (b) $r_{s}=0.085$.

quantum phase transition, is offered in Sec. V, based on the study of the superfluid properties of the system.

\section{SUPERFLUIDITY AND CONDENSATION}

Figure 6 shows a typical result for the superfluid and condensate fractions of a dipolar assembly. In this particular case, the number of particles $N$ is 100 , and the trap strength yields a value of $r_{s}$ in the middle of the trap approximately equal to 0.22 , as a function of temperature. As the temperature is lowered below $T^{*} \lesssim 1$, both the superfluid and condensate fractions become nonzero, saturating to very different values in the $T^{\star} \rightarrow 0$ limit. Specifically, the superfluid fraction $\rho_{s}$ approaches unity, whereas the condensate fraction $\rho_{c}$ is less than $20 \%$ at $T^{\star}=0$. On the other hand, in the large- $r_{s}$ limit (weaker trap strength) superfluid and condensate fractions are close in magnitude, both close to unity at low temperature, as expected for a more dilute system. That interparticle interactions have a stronger (depleting) effect on the condensate than 


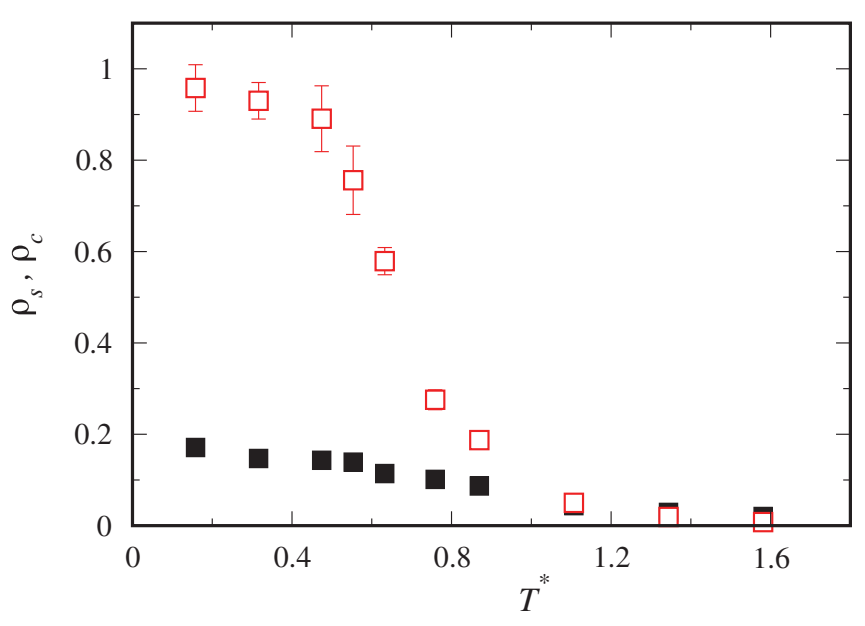

FIG. 6. (Color online) Superfluid fraction $\rho_{s}$ (red open squares) and condensate fraction $\rho_{c}$ (black filled squares) as function of reduced temperature for system with $N=100$ and trap strength yielding a value of the inter-particle distance $r_{s}=0.22$ in the center of the trap.

the superfluid fraction is of course not a novel feature of this many-body system. The same trend is predicted theoretically ${ }^{37}$ and observed experimentally ${ }^{38}$ in condensed helium.

It is well understood theoretically that SF and BEC are both underlain by quantum-mechanical exchanges of identical particles. ${ }^{39}$ In order to explore this notion in greater detail, in Fig. 7 we show the relative frequency of occurrence of exchange cycles involving a variable number $1 \leqslant n \leqslant N$ of particles (here, $N=100$ ). These results are for four different values of the interparticle distance in the center of the trap, namely, $r_{s}=0.94,0.36,0.14,0.09$ at low temperature. In all four cases, the superfluid fraction is $100 \%$, (within statistical uncertainties), whereas the condensate fraction goes from $54 \%$ for the the most dilute to just $6 \%$ (i.e., a mere six particles) for the densest assembly. This difference in the fraction of the system condensed to the same single-particle quantum-mechanical state is directly related to the much greater frequency with which permutation cycles (of any length) occur at lower density, i.e., with weaker interactions.

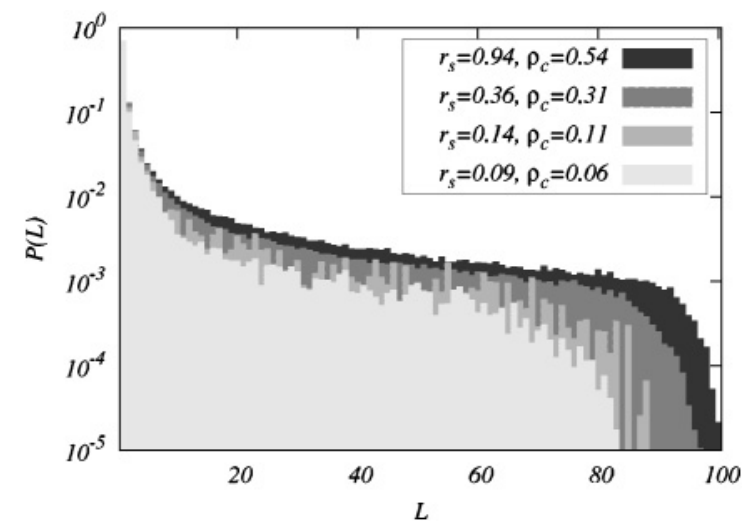

FIG. 7. Relative frequency of permutation cycles of length $L$ for simulations with four different trap strengths corresponding to $r_{s}=0.94,0.36,0.14,0.09$ and for $N=100$ and temperature $T^{*}=0.1$, at which the system is essentially in the ground state.
At the same time, these results clearly show that there is no obvious, direct numerical connection between the length of the longest observed exchange cycle, or the statistics of occurrence of cycles comprising specific numbers of particles, and the superfluid fraction. Remarkably, the superfluid signal in the low-temperature limit is unaffected by the significant differences in the statistics of exchange cycles at the four thermodynamic conditions considered.

As explained in Sec. II, we can compute the local superfluid and condensate densities, gaining greater insight into the relationship between SF and BEC. Figure 8 shows the total, superfluid, and condensate radial density profiles, for a system of $N=100$ particles and two different values of interparticle separation $r_{s}$ in the center of the trap. The system is essentially in the ground state, i.e., the superfluid fraction $\rho_{s} \sim 1$ in both cases. As one can see, the condensate density becomes suppressed relative to the superfluid one, as the interparticle separation is decreased. Significantly, however, both local
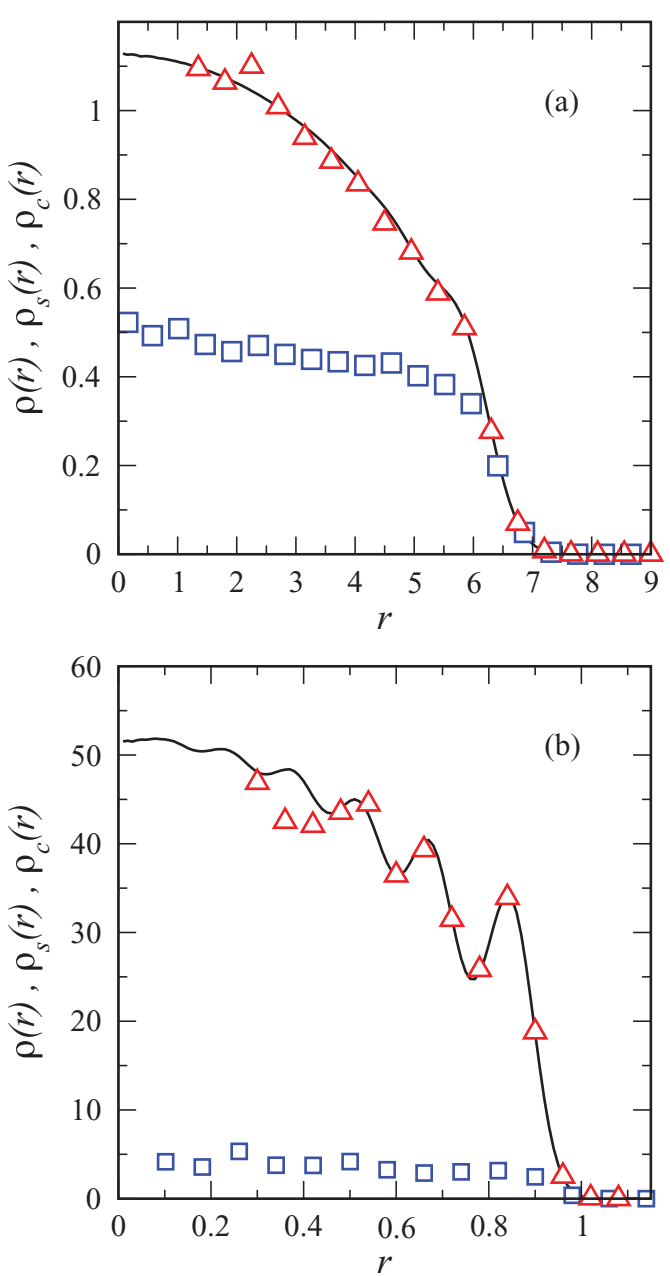

FIG. 8. (Color online) Total $\left[\rho(r)\right.$, line], condensate $\left[\rho_{c}(r)\right.$, squares], and superfluid $\left[\rho_{s}(r)\right.$, triangles] radial density profiles, for a system with $N=100$ dipolar bosons for two different trap strengths, yielding values of the interparticle distance $r_{s}$ in the center equal to 0.94 and 0.14 . The system is in the ground state and the overall superfluid fraction is near unity in both cases. Statistical and systematic uncertainties are estimated to be of the order of the sizes of the symbols. 
quantities are almost uniformly distributed throughout the system. For the superfluid density, this is consistent with recent findings for parahydrogen clusters. ${ }^{40}$ The condensate density displays a markedly different behavior in the vicinity of the surface, depending on the degree of particle localization. At the largest average interparticle separation (lowest density), the system near the surface is highly dilute, and the local condensate fraction approaches $100 \%$, as observed in helium droplets as well. ${ }^{23}$ As the density of the system is increased, particles near the surface become increasingly localized, and BEC is locally suppressed, while remaining finite near the center of the trap, where the physical behavior is that of a superfluid.

It is worth noting that the behavior of the system of dipolar bosons studied here deviates significantly from that of hardcore bosons studied previously by DuBois and Glyde. ${ }^{41}$ In that study, it was found that the local condensate density becomes severely depleted at the center of the trap, as the density is increased. In particular, the condensate forms a shell at the edge of the trap for strongly interacting systems. This effect is absent here, a fact that can presumably be attributed to the long-range nature of the dipole-dipole interaction, which in turn renders the use of any local density approximation questionable.

As mentioned above and as will be quantitatively discussed in the next section, on decreasing the average interparticle separation a crystalline phase eventually nucleates in the center of the trap, at which point both superfluidity and condensation become suppressed in the system. The absence of a sharp phase transition in a finite system results in a value of the superfluid fraction less than 1 in the ground state, as superfluid and crystalline phases coexist. In the next section, we determine the ground-state phase diagram of the system by calculating the superfluid and condensate fractions as a function of system size and interparticle separation.

\section{GROUND-STATE PHASE DIAGRAM}

As discussed in Sec. I, a $T=0$ (quantum) phase transition between a crystalline, insulating phase and a superfluid phase is observed in the bulk system, driven by the chemical potential (i.e., the density). One of the issues of interest here is whether any remnant of such a transition can be observed, by either simulation or possible experiments, in a finite system. In Fig. 9 (top), we show values of the superfluid fraction $\rho_{s}$ corresponding to different interparticle distances $r_{s}$ in the middle of the trap. These values are computed for various system sizes (between $N=20$ and 200), at a temperature sufficiently low for them to be regarded as ground-state estimates.

Although no sharp phase transition can be observed in a finite system, the data clearly show a crossover between two regimes, a superfluid $\left(\rho_{s} \sim 1\right)$ and crystal $\left(\rho_{s} \sim 0\right)$, taking place roughly in the same region of $r_{s}$ in which it is observed in numerical studies of the ground-state bulk phase diagram ${ }^{16}$ (shaded region of Fig. 9). It is worth mentioning that, in the range $0.067 \lesssim r_{s} \lesssim 0.12$, during a sufficiently long simulation, the system switches back and forth between two different regimes, characterized by zero and finite superfluid density. This behavior, already reported in previous work, ${ }^{42}$ is not

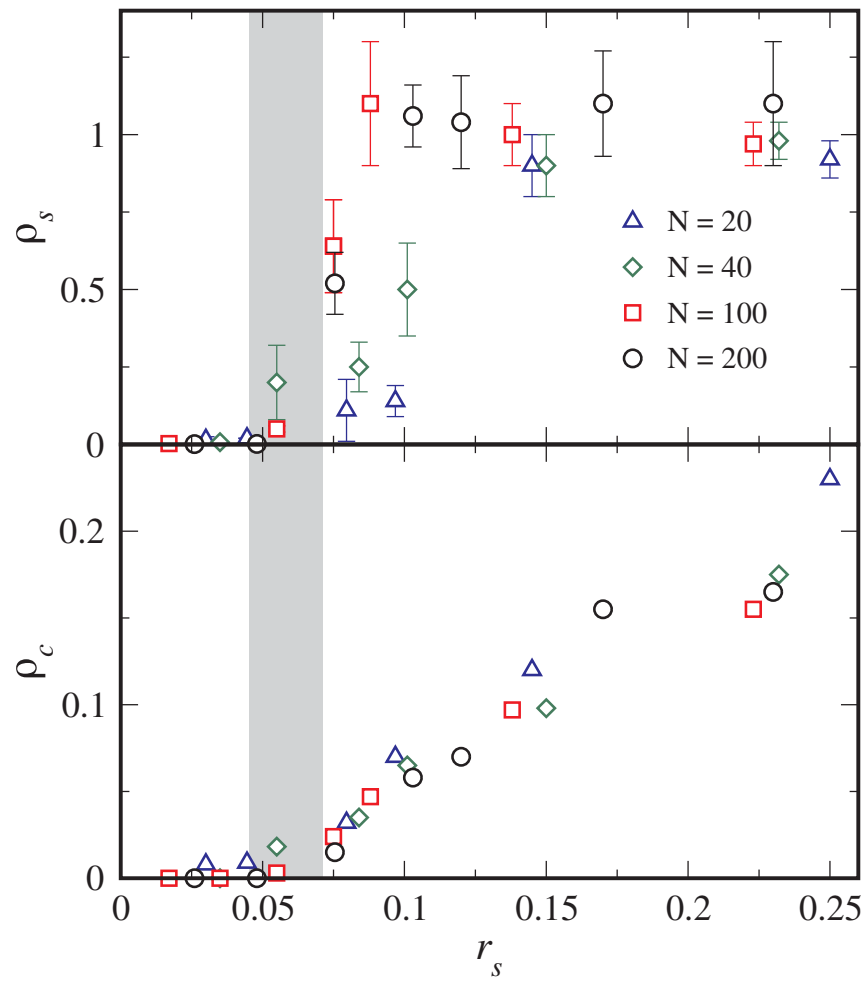

FIG. 9. (Color online) Top: Superfluid fraction (extrapolated to $T \rightarrow 0)$ vs interparticle distance $r_{s}$ for different system sizes. Bottom: Condensate fraction $\rho_{c}$ as defined in the text (extrapolated to $T \rightarrow 0$ ) vs interparticle distance $r_{s}$ for different system sizes. We have offset the condensate value by $1 / N$, namely, the smallest possible eigenvalue of the one-body density matrix. The shaded region represents the estimated range for the critical value of $r_{s}$ predicted for the homogeneous system from Ref. 16.

unlike what is observed in small clusters of parahydrogen; ${ }^{29,30}$ it suggests that the two phases are energetically very close, and in practice renders lengthy simulations necessary, in order to assign a reliable value to the superfluid fraction. This is the reason for the (relatively) large statistical uncertainties of some of the estimates reported in Fig. 9.

The results in Fig. 9 (top) suggest that melting and freezing values for $r_{s}$ at $T=0$ should lie in the range $0.055 \leqslant r_{s} \leqslant$ 0.07, which is consistent with bulk studies. (In particular, in two separate quantum Monte Carlo studies of the bulk system the transition was predicted to occur either in the region $0.045 \leqslant r_{s} \leqslant 0.071,{ }^{16}$ or in the region $0.056 \leqslant r_{s} \leqslant 0.062,{ }^{17}$ in the latter case by extrapolating the system energy to the thermodynamic limit.) A precise determination of the coexistence region is obviously beyond the scope of what can be achieved in a study of a finite system.

Figure 9 (bottom) shows the condensate fraction $\rho_{c}$ for the same set of simulation parameters for which the superfluid fraction is given in the top part. Here, we define "condensate fraction" $\rho_{c}=N_{0} / N$, i.e., the largest eigenvalue $N_{0}$ of the cylindrically averaged one-particle density matrix, divided by the total number $N$ of particles. In order to facilitate the comparison of results obtained for systems of different size, we have subtracted $1 / N$ from all estimates of $\rho_{c}$, as 1 is the 
lowest possible value of $N_{0}$. Such a correction is nontrivial for the smallest systems.

In the thermodynamic limit, $\rho_{c}=0$ at any finite temperature, as thermal fluctuations destroy off-diagonal long-range order. In a finite system, however, $\rho_{c}$ can be finite, and indeed it is found to be finite for values of $r_{s}$ above $\sim 0.07$, i.e., in the superfluid region. Remarkably, the data in Fig. 9 (bottom) are consistent with the vanishing of $\rho_{c}$ concurrently with that of the superfluid fraction $\rho_{s}$, as the system is compressed to the crystalline phase.

In an infinite system, the single-particle state in which bosons condense is a plane wave of momentum $\mathbf{k}=\mathbf{0}$, simply based on considerations of translational invariance. This results in a (theoretical) $\delta$-like peak at $\mathbf{k}=\mathbf{0}$ in $n(\mathbf{k})$. Experimental evidence of phase coherence throughout the system can be detected in the $n(\mathbf{k})$ of a spatially confined system as well, through the appearance of a peak at $\mathbf{k}=\mathbf{0}$ at low temperature, even though harmonic confinement has the effect of broadening it, its width being of order $1 / L$.

In experiments with trapped cold atoms, the momentum distribution is routinely measured via time-of-flight absorption imaging. As a guide for possible experiments, it is therefore instructive to compute the momentum distribution in the limit $T^{*} \rightarrow 0$, for two systems which have a superfluid and a crystalline ground state.

For a two-dimensional cylindrically symmetric system, the momentum distribution is given by

$$
n(k)=\int_{0}^{\infty} d r r \tilde{n}(r) J_{0}(k r),
$$

where $\tilde{n}(r)$ is the spatially averaged one-body density matrix, computed by simulation, and $J_{0}$ is the zeroth-order Bessel function. ${ }^{43}$ Figure 10 shows the computed $n(k)$ for a system of $N=200$ dipolar bosons at three different temperatures, for a

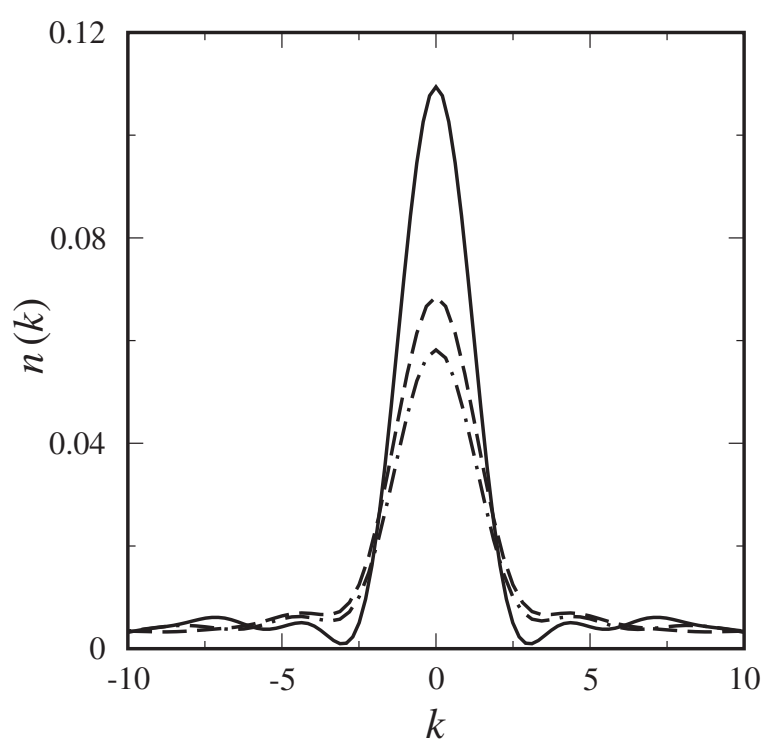

FIG. 10. Momentum distribution $n(k)$ for an assembly of $N=200$ dipolar bosons in a two-dimensional harmonic trap. The strength of the trap is such that the interparticle distance at its center is $r_{s}=0.122$. Results shown are for temperatures $T=0.05$ (solid line), 0.2 (dashed line), and 0.4 (dot-dashed line), all in units of $T^{\star}$, defined in the text. trap strength yielding a value of $r_{s} \sim 0.122$ in the middle of the trap, i.e., well into the superfluid region of the phase diagram. The development of a sharp peak at $k=0$ as the temperature is lowered is clear; the value of the superfluid fraction at low $T$ approaches $100 \%$ in this case. No evidence of such a peak is observed in our simulations for values of $r_{s}$ for which the assembly of dipolar particles does not display a measurable superfluid response.

Thus, the results of this study suggest that much of the physics of the crystal-superfluid transition in dipolar bosons can be probed experimentally on a system comprising as few as several hundred particles.

\section{DISCUSSION AND CONCLUSION}

We have investigated by extensive quantum Monte Carlo simulations the properties of dipolar bosons confined in two-dimensional traps. In particular, we have analyzed the structure of the system, superfluidity, and Bose-Einstein condensation, as well as the ground-state phase diagram for the Hamiltonian (1) for different system sizes and as a function of in-trap density. We have considered system sizes up to $N=$ 1000, a number relevant to current and planned experiments. It is therefore our expectation that a direct comparison between theory and experiment should be possible.

We find that the physics of the system in the middle of the trap closely mimics that expected in an infinite system, at least in the region of density wherein the superfluid-insulator quantum phase transition is expected, if the number of particles in the trap is of the order of a few hundreds. In particular, a transition between superfluid and crystal-like phases is evident, as shown by our results for the density profiles and local pair correlation function as well as the superfluid and condensate fractions. By considering system sizes up to $N=1000$, we verify that such a transition occurs for an interparticle separation of $0.055 \leqslant r_{s} \leqslant 0.07$, which is consistent with the most reliable theoretical predictions for the bulk case. $^{16}$

In order to gain deeper understanding of the elusive relationship between superfluidity and Bose-Einstein condensation, a study of the global and local superfluid properties has also been performed. The general conclusion is consistent with that obtained for systems with different interactions, namely, that strong interparticle interactions have a much more pronounced quantitative effect on the condensate than on the superfluid fraction. However, our results are consistent with the concurrent disappearance of both as the system freezes at high density. With the exception of a narrow region near the edge of the trap, where BEC is either enhanced or suppressed depending on particle localization, the superfluid and condensate density are largely uniform throughout the system.

In striking contrast with the case of hard-core bosons, ${ }^{41}$ BEC is not depressed at the center of the trap in the presence of strong interactions (i.e., at high density). Such a qualitative difference points to the importance of the long-range nature of the interactions in (1), with the inference that a local density approximation cannot be straightforwardly applied here.

We have also investigated the momentum distribution as a function of temperature. The presence of coherence at low 
temperature, if the system features a superfluid ground state, is rendered evident by the appearance of a pronounced peak at zero momentum, in the low-temperature limit. This seems to indicate that important signatures of a quantum phase transition predicted in the bulk system, should be readily observed in current or planned experiments.

\section{ACKNOWLEDGMENTS}

The authors thank Fabio Mezzacapo for useful discussions. This work was supported in part by the Natural Science and Engineering Research Council of Canada under Research Grant No. 121210893, and by the Alberta Informatics Circle of Research Excellence (iCore). *jain@ualberta.ca

†cinti@ualberta.ca

${ }^{1}$ A. J. Leggett, Quantum Liquids (Oxford University Press, Oxford, 2006).

${ }^{2}$ R. P. Feynman, Phys. Rev. 91, 1291 (1953).

${ }^{3}$ K. B. Davis, M. O. Mewes, M. R. Andrews, N. J. van Druten, D. S. Durfee, D. M. Kurn, and W. Ketterle, Phys. Rev. Lett. 75, 3969 (1995).

${ }^{4}$ M. Anderson, J. Ensher, M. Matthews, C. Wieman, and E. Cornell, Science 269, 198 (1995).

${ }^{5}$ W. Krauth, Phys. Rev. Lett. 77, 3695 (1996).

${ }^{6}$ S. Heinrichs and W. Mullin, J. Low Temp. Phys. 113, 231 (1998).

${ }^{7}$ S. Moroni and M. Boninsegni, J. Low Temp. Phys. 136, 129 (2004).

${ }^{8}$ M. Boninsegni, N. V. Prokof'ev, and B. V. Svistunov, Phys. Rev. E 74, 036701 (2006).

${ }^{9}$ R. T. Azuah, W. G. Stirling, H. R. Glyde, M. Boninsegni, P. E. Sokol, and S. M. Bennington, Phys. Rev. B 56, 14620 (1997).

${ }^{10}$ H. R. Glyde, R. T. Azuah, and W. G. Stirling, Phys. Rev. B 62, 14337 (2000).

${ }^{11}$ A. Griesmaier, J. Werner, S. Hensler, J. Stuhler, and T. Pfau, Phys. Rev. Lett. 94, 160401 (2005).

${ }^{12}$ T. Lahaye, C. Menotti, L. Santos, M. Lewenstein, and T. Pfau, Rep. Prog. Phys. 72, 126401 (2009).

${ }^{13}$ P. Jain and M. Boninsegni, Phys. Rev. A 83, 023602 (2011).

${ }^{14}$ W. R. Anderson, J. R. Veale, and T. F. Gallagher, Phys. Rev. Lett. 80, 249 (1998).

${ }^{15}$ G. Pupillo, A. Micheli, M. Boninsegni, I. Lesanovsky, and P. Zoller, Phys. Rev. Lett. 104, 223002 (2010).

${ }^{16}$ H. P. Büchler, E. Demler, M. Lukin, A. Micheli, N. Prokof'ev, G. Pupillo, and P. Zoller, Phys. Rev. Lett. 98, 060404 (2007).

${ }^{17}$ G. E. Astrakharchik, J. Boronat, I. L. Kurbakov, and Y. E. Lozovik, Phys. Rev. Lett. 98, 060405 (2007).

${ }^{18}$ C. Mora, O. Parcollet, and X. Waintal, Phys. Rev. B 76, 064511 (2007).

${ }^{19}$ B. Spivak and S. A. Kivelson, Phys. Rev. B 70, 155114 (2004).

${ }^{20}$ A. Filinov, N. V. Prokof'ev, and M. Bonitz, Phys. Rev. Lett. 105, 070401 (2010).
${ }^{21}$ I. L. Kurbakov, Y. E. Lozovik, G. E. Astrakharchik, and J. Boronat, Phys. Rev. B 82, 014508 (2010).

${ }^{22}$ F. Cinti, P. Jain, M. Boninsegni, A. Micheli, P. Zoller, and G. Pupillo, Phys. Rev. Lett. 105, 135301 (2010).

${ }^{23}$ D. S. Lewart, V. R. Pandharipande, and S. C. Pieper, Phys. Rev. B 37, 4950 (1988).

${ }^{24}$ P. Sindzingre, M. L. Klein, and D. M. Ceperley, Phys. Rev. Lett. 63, 1601 (1989).

${ }^{25}$ V. Bagnato and D. Kleppner, Phys. Rev. A 44, 7439 (1991).

${ }^{26}$ Z. Hadzibabic, P. Kruger, M. Cheneau, S. P. Rath, and J. Dalibard, New J. Phys. 10, 045006 (2008).

${ }^{27}$ Y. Lozovik, S. Volkov, and M. Willander, JETP Lett. 79, 473 (2004).

${ }^{28}$ M. Boninsegni, N. Prokof'ev, and B. Svistunov, Phys. Rev. Lett. 96, 070601 (2006).

${ }^{29}$ F. Mezzacapo and M. Boninsegni, Phys. Rev. Lett. 97, 045301 (2006).

${ }^{30}$ F. Mezzacapo and M. Boninsegni, Phys. Rev. A 75, 033201 (2007).

${ }^{31}$ J. E. Cuervo, P.-N. Roy, and M. Boninsegni, J. Chem. Phys. 122, 114504 (2005).

${ }^{32}$ Y. Kwon, F. Paesani, and K. B. Whaley, Phys. Rev. B 74, 174522 (2006).

${ }^{33}$ P.-O. Löwdin, Phys. Rev. 97, 1474 (1955).

${ }^{34}$ L. Onsager and O. Penrose, Phys. Rev. 104, 576 (1956).

${ }^{35}$ The statement made in Ref. 5, that the number of particles in the condensate corresponds to the length of the longest observed exchange cycle, is incorrect.

${ }^{36}$ M. E. Fisher and A. N. Berker, Phys. Rev. B 26, 2507 (1982).

${ }^{37}$ S. Moroni and M. Boninsegni, J. Low Temp. Phys. 136, 129 (2004).

${ }^{38}$ H. R. Glyde, S. O. Diallo, R. T. Azuah, O. Kirichek, and J. W. Taylor, Phys. Rev. B 83, 100507 (2011).

${ }^{39}$ D. M. Ceperley, Rev. Mod. Phys. 67, 279 (1995).

${ }^{40}$ F. Mezzacapo and M. Boninsegni, Phys. Rev. Lett. 100, 145301 (2008).

${ }^{41}$ J. L. DuBois and H. R. Glyde, Phys. Rev. A 63, 023602 (2001).

${ }^{42}$ J. Böning, A. Filinov, P. Ludwig, H. Baumgartner, M. Bonitz, and Y. E. Lozovik, Phys. Rev. Lett. 100, 113401 (2008).

${ }^{43}$ M. Boninsegni, Phys. Rev. B 79, 174203 (2009). 